\title{
The Measurement and Evaluation of Standard DQE in Digital Radiography
}

\author{
Luxiang ${ }^{1, a^{*}}$, Zhang jun-yi ${ }^{2, b}$ \\ ${ }^{1}$ Beijing Institute of Metrology, China \\ 2 Direct Digital Imaging Technology (Beijing) INC. \\ a luxiang@bjjl.cn, ${ }^{\mathrm{b}}$ zhjy1999@gmail.com
}

\begin{abstract}
This study introduces the measurement and evaluation of the standard detective quantum efficiency in digital radiography. The detective quantum efficiency (DQE) is the most suitable parameter for describing the imaging performance of digital Radiography. DQE is defined as the squared ratio of the signal-to-noise ratio at the output to the signal-to- noise ratio at the input of digital radiography. In this study, the main work is to use some device to gain images, which used to obtain the modulation transfer function (MTF) and Noise Power Spectrum (NPS), according to the international standard IEC 62220-1. We also do some work about the algorithm of MTF and NPS. Finally we design a set of DQE assessment method which can be used in metrology and measurement of digital radiography. We define the test conditions as follows: RQA5 radiation quality, $21 \mathrm{mmAl}$ additional filtration. Experiment proved that DQE can show the basic properties of the flat panel detector in DR system. In the process of obtaining the DQE, the key performance parameters could be measured and normalized, which includes DQE, MTF, detector response curve and NPS. The digital radiography with poor DQE has the low imaging quality. The patients should have more body surface irradiation dose. Metrological verification and quality control personnel can determine the working station of DR system according to the indexes of DQE various performance indicators, which is good for decreasing collective effective dose equivalent.
\end{abstract}

Keywords-Digital radiography, Flat panel detector, Detective quantum efficiency, Medical images measuring

\section{INTRODUCTION}

Digital radiography (DR) is a famous breakthrough in the field of X-ray radiation. Since it has many advantages than conventional screen-film system and computed radiography, such as more functions, higher efficiency, lower exposure dose and higher image quality, its market penetration raised rapidly in recent years. One of the core components in DR is Flat panel detector (FPD), which absorbs the incoming X-rays and produces output electrical signals and then transforms the signals to digital image. The Characteristics of FPD highly influence the imaging properties of a DR system. Therefore, Metrological verification is needed. At present, there are two steps can do DR metrological verification. One step is judging the dose deviation between nominal dose of a system and a measured value, the other step is distinguishing line pair or different contrast in the image of a special phantom by eyes. The last type cannot reflecting the real characteristic of the system because the progress of converting $X$ rays to image is not considered and human factors is not very certain. Furthermore, this method cannot monitor system running. In order to solve these problems, the connection between images obtained from FPD with the X-rays emitted from tube should be considered. Normally, DQE is used to describe the imaging performance of an X-ray imaging device[1,2,3,4].

\section{METHOD}

The DQE is the most suitable parameter for describing the imaging performance of digital Radiography. DQE is defined as the squared ratio of the signal-to-noise ratio at the output to the signal-to- noise ratio at the input of digital radiography. For digital radiography, the input is the $\mathrm{X}$-ray photons, and the output is the digital image. It is well known that the $\mathrm{X}$-ray photons depend on the X-ray exposure dose with the radiation quality. DQE reflects the relationship between the X-ray dose and the image form the flat-panel detector. This could be achieved by traditional metrology and measurement technique used in digital radiography. The equation for the frequency-dependent DQE is:

$$
D Q E(\mu, v)=S N R_{\text {out }}^{2} / S N R_{\text {in }}^{2}
$$

In formula $1, \mu$ and $v$ mean the spatial frequencies in the $\mathrm{x}$ and $\mathrm{y}$ direction; SNRin and SNRout are the input and output Signal-to-Noise ratios, respectively.

\section{A SNRin}

SNRin can be calculated by:

$S_{N N R_{i n}^{2}}^{2}=w_{\text {in }}(\mu, v)$

Where win $(\mu, v)$ equals to the X-ray photo fluency $\mathrm{Q}$, which can be calculated by

$$
w_{\text {in }}(\mu, v)=Q=K_{a} \int \phi(E) / K_{a} d E=K_{a} S N R^{2}
$$

Where $\mathrm{E}$ is the energy of $\mathrm{X}$-ray energy; $\mathrm{Ka}$ is the measured air kerma, unit: $\mu \mathrm{Gy} ; \Phi(\mathrm{E})$ is the flux per air kerma; SNR2is the squared signal-to-Noise ratio per air kerma. During a test, the radiation qualities should be 
TABLE I RAQ5 PARAMETER

\begin{tabular}{|c|c|c|c|c|}
\hline $\begin{array}{l}\text { Radiation } \\
\text { quality No. }\end{array}$ & $\begin{array}{l}\text { Approximate } \\
\text { X-Ray tube } \\
\text { Voltage } \\
\mathrm{kV}\end{array}$ & $\begin{array}{l}\text { HALF-VALUE } \\
\text { layer(HVL) } \\
\mathrm{mm} \mathrm{Al}\end{array}$ & $\begin{array}{l}\text { Additional filtration } \\
\mathrm{mm} \mathrm{Al}\end{array}$ & $\begin{array}{c}S_{N R^{2}}^{2} \\
1 /\left(\mathrm{mm}^{2} \cdot \mu \mathrm{Gy}\right)\end{array}$ \\
\hline RQA5 & 70 & 7.1 & 21 & 30174 \\
\hline
\end{tabular}

\section{B SNRout}

SNRout can be calculated by:

(4)

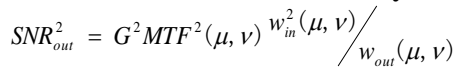

In formula $4, \mathrm{G}$ is the gain of the detector at zero spatial frequency. When the pixel value is exposure quantum per unit area calculated by the liner correlation between quantum with raw data and does, $G$ can be omitted. $\operatorname{MTF}(\mu, v)$ is the pre-sampling modulation transfer function of the FPD. MTF function reflects the sharpness of DR system, which is a measure of the ability of DR system reproducing image contrast from subject contrast. Mathematically, the MTF is a plot of the radio of the output-to-input single -frequency sinusoidal function of their spatial frequency. The method of obtaining MTF function can be calculated as follows $[6,7,8]$ :

(1) Obtain an irradiation image of tungsten edge device according to IEC 62220-1

(2) Analysis the image and get an edge profile

(3) Construct an oversampled edge spread function (ESF)

(4) The oversampled ESF is differentiated smoothed and its digital Fourier transform is calculated. The modulus of this Fourier transform yields the MTF.

Wout $(u, v)$ is the NPS of the output image. In radiographic imaging, noise interferes with the visualization of an abnormality of interest and with the interpretation of an image. Radiographic noise is best characterized by its NPS $[9,10]$. The NPS is the variance of noise within an image divided among various spatial frequency components of the image. Mathematically, the NPS is the normalized squares of Fourier amplitudes averaged over an ensemble of noisy but otherwise uniform images.

The NPS at the output of the digital X-ray imaging device (Wout $(\mathrm{u}, \mathrm{v})$ ) can be calculated according to this standard is:

$$
W_{\text {out }}(u, v)=\left.\lim _{M \rightarrow \infty} \frac{\Delta x \Delta y}{\mathrm{M} \cdot 256 \cdot 256} \sum_{m=1}^{M} \sum_{i=1}^{256} \sum_{j=1}^{256}\left(I\left(x_{i}, y_{j}\right)-S\left(x_{i}, y_{j}\right)\right) \exp \left(-2 \pi i\left(\mu x_{i}+y_{j}\right)\right)\right|^{2}
$$

In formula $5, \mathrm{I}(\mathrm{xi}, \mathrm{yj})$ is the region of interest (ROI) with a size of $256 \times 256$; $M$ is the number of the ROIs; $\mathrm{S}(\mathrm{xi}, \mathrm{yj})$ is reference image which can be calculated by averaging several images.

\section{C $D Q E$}

According to formula 2, formula 3, formula 4and formula5, DQE can be calculated by:

$$
D Q E(u, v)=G^{2} \operatorname{MTF}^{2}(\mu, v) W_{\text {in }}(u, v) / W_{\text {our }}(u, v)
$$

(6)

During the measurement of DQE, the dose of the input irradiation, the MTF of the detector of DR, the Noise of the image and the liner relation between raw data with input air kerma are all measured, thus it is a comprehensive verification. A system with higher DQE can obtain good image with lower irradiation, and the false positive or negative of identify lesions can be reduced.

\section{RESULTS}

\section{A $\quad M T F$}

In clinic, the spatial resolution is very important, because only lesions larger than special frequency can be recognized. People use line pair card to test spatial resolution for a long time, but it highly rely on eyes, thus it is not very certain. It test of DQE, the spatial resolution can be obtained by MTF. In this study, we choice one type of FPD to calcite the result of DQE, and the size of the FPD is $35 \mathrm{~cm} \times 43 \mathrm{~cm}$, and the active matrix is $2560 \times 3072$.

Fig .1-3 shows the MTF results under irradiation of RQA5, 40ms and 100mA. 


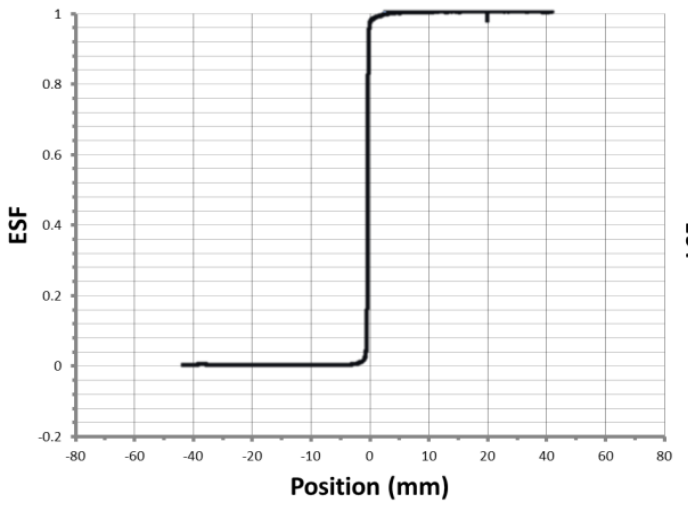

Figure 1 Edge-spread function

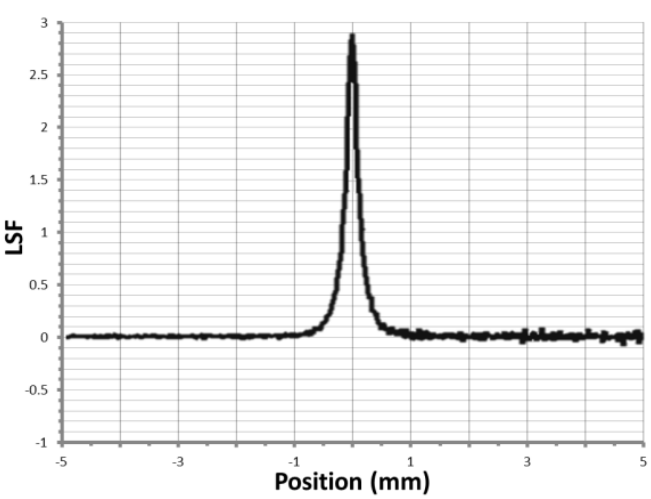

Figure 2 Line-Spread function

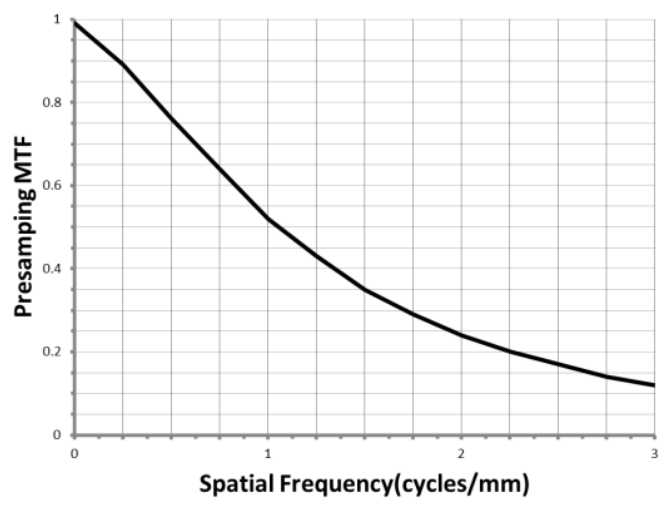

Figure 3 MTF value diagram

\section{B $D Q E$}

The DQE of the system in 3.3 was also tested, and the result is shown in Fig .4. When a lesion is recognize in an image, it can be confirm by considering corresponding DQE value. Quality inspectors can judge a system by DQE. The result also shows that the efficiency of the X-ray conversion decrease with spatial resolution.

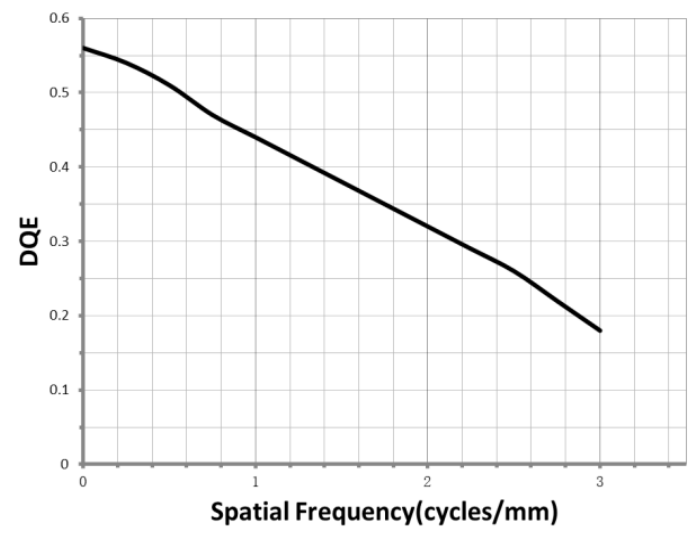

Figure 4 DQE value diagra

Table2 shows the MTF and DQE value corresponding to different spatial resolution. 
TABLE II THE MTF AND DQE VALUE BASED ON SPATIAL FREQUENCY

\begin{tabular}{ccc}
\hline $\begin{array}{c}\text { Spatial frequency } \\
\text { (cycles/mm) }\end{array}$ & MTF value & DQE value \\
\hline 0.25 & 0.89 & 0.54 \\
0.50 & 0.76 & 0.51 \\
0.75 & 0.64 & 0.47 \\
1.00 & 0.52 & 0.44 \\
1.25 & 0.43 & 0.41 \\
1.50 & 0.35 & 0.38 \\
1.75 & 0.29 & 0.35 \\
2.00 & 0.24 & 0.32 \\
2.25 & 0.20 & 0.29 \\
2.50 & 0.17 & 0.26 \\
2.75 & 0.14 & 0.22 \\
3.00 & 0.12 & 0.18 \\
\hline
\end{tabular}

From table2, we can conclude MTF and DQE values have a trend of relationship with the spatial frequency. With the increase of spatial frequency, MTF and DQE gradually reduced.

MTF, which comprehensively reflects the image contrast and spatial resolution, can be used as a simple image contains information of evaluation index. For DR digital imaging, image quality in addition to the detector unit area, the factors such as sensitivity are affected by image processing method, the influence of the incident X-ray dose and energy. So, using the MTF has not fully expressed the quality of digital images. Now some adopt more perfect DQE index to evaluate the quality of digital image, because it combines various parameters affecting the quality of images, including the MTF. Therefore, performing DQE measurement is further perfection and development of image quality evaluation method.

\section{SUMMARY}

For medical image, we want to see the quality of the image and think about the conditions of the imaging. Only for images, the image quality should be evaluated by the spatial resolution and contrast resolution, dynamic range, the MTF, noise and other indicators. For digital imaging system, the indicators in addition to the detector unit area, the factors such as sensitivity, are affected by image processing method, the influence of the incident X-ray dose and energy, any distributed parameter cannot fully explain the characteristics of the DR system. The technology about more lower the incident X-ray dose for more higher SNR of images represent the level of detector performance. Currently the DR system manufacturers generally used DQE as evaluation index. The DQE index combination of the image contrast spatial resolution, noise and incident X-ray dose, and it can be a comprehensive ability expression of digital imaging system to detection of contrast. The DQE index is considered to be the evaluation criteria of digital radiography for the imaging quality.

\section{ACKNOWLEDGEMENT}

This project is supported by Special Fund for AQSIQ-Scientific Research in the Public Interest (201410111).

\section{REFERENCES}

[1] N. Ulrich and G.K. Susanne. Determination of the detective quantum efficiency of a digital x-ray detector: Comparison of three evaluations using a common image data set. Med. Phys.2004,31(8):2205-2211

[2]Y. Lin,H. Luo,J.T. DobinIII, et al An image-based technique to assess the perceptual quality of clinical chest radiographs. Med. Phys.2012,39(11):7019-7031

[3] E.A. Schnell, E. Samei,J.T. Dobbins, Plate-specific gain map correction for the improvement of detective quantum efficiency in computed radiography. Med. Phys.2012,39(3):1495-1504

[4] J.G.Choi ,Y.S. Kim, H.S. Park, Evaluation of the Clinical Performance by Using the Effective DQE for a Prototype Digital Breast Tomosynthesis System, Journal of the Korean Physical Society 2012,60(5):869-874.

[5]IEC 62220-1, Medical electrical equipment -Characteristics of digital X-ray imaging devices-Part 1:Determination of the detective quantum efficiency. 2003.

[6] E. Samei, M.J. Flynn, D.A Reimann, A method for measuring the presampled MTF of digital radiographic systems using an edge test device[J]. Med Phys 1998, 25(1):102-113.

[7] E. Samei, J.F. Michael, G.C. Harrell,et al, DQE of direct and indirect digital radiographic systems. Medical Imaging 2001: Physics of Medical Imaging. Proceedings of SPIE, 2001, 189-197.

[8]E. Samei,T.R. Nicole,T.D. James, et al. Intercomparison of methods for image quality characterization. I. modulation transfer function. Med. Phys., 2006, 33(5): 1454-1465.

[9] E. Samei, Performance of digital radiographic detectors: quantification and assessment methods. Advances in digital radiography: RSNA 2003: Categorical course in diagnostic Radiology Physics, 2003, 37-47

[10] F. Gao, H. Zhao,L. Zhang, et al. Techniques to improve the accuracy of noise power spectrum measurements in digital X-ray imaging based on background trends removal, Med. Phys.,2011,38(3):1600-1610 